\title{
Degradabilidad ruminal de la alfalfa en la zona oriental del Valle Central de Costa Rica ${ }^{1}$
}

\section{Ruminal degradability of alfalfa in the Costa Rica's Central Valley, Eastern zone}

\author{
Carlos Boschini-Figueroa ${ }^{2}$,Pablo Chacón-Hernández ${ }^{2}$
}

\begin{abstract}
Resumen
El objetivo del trabajo fue evaluar en bovinos de leche, el potencial de degradabilidad ruminal de cuatro variedades de alfalfa (Medicago sativa) desarrolladas en la República Argentina. La investigación se llevó a cabo en la Estación Experimental Alfredo Volio Mata, localizada en la provincia de Cartago, Costa Rica; la zona presenta una temperatura promedio de $17,9^{\circ} \mathrm{C}$, una altura de $1542 \mathrm{msnm}$ y precipitación de 1465,9 mm anuales distribuidos principalmente de mayo a noviembre. El muestreo fue realizado durante la época lluviosa del año 2014, por medio de la cosecha de alfalfa, a nivel del suelo, de muestras de las variedades Mora, Patricia, Victoria y Súper Monarca, a edades de cosecha de 28, 35, 42, 49, 56 y 63 días de rebrote; todas las muestras fueron secadas, molidas e incubadas por duplicado en dos vacas Jersey-Reyna fistuladas ruminalmente, por 0, 3, 6, 12, 24 y 48 horas. Los resultados fueron analizados mediante el algoritmo de Marquardt con el programa estadístico SAS. Solo hubo diferencias estadísticamente significativas $(\mathrm{p}<0,05)$ para las fracciones solubles y la tasa de degradación según la edad de cosecha, con valores de fracción soluble, potencialmente degradable y tasa de degradación entre 35,34\%-44,65\%,36,46\%-39,96\% y 0,0734/h-0,1176/h, respectivamente. Asimismo, se obtuvieron porcentajes de degradabilidad efectiva entre 59,06\%-67,09\%, con una tasa de paso de $6 \% / \mathrm{h}$, según el tiempo de rebrote. Según los resultados obtenidos, las variedades analizadas de $M$. sativa mostraron un forraje digestible y con potencial para uso en animales altamente productivos.
\end{abstract}

Palabras clave: Medicago sativa, nutrición de rumiantes, digestibilidad in situ.

\begin{abstract}
The objective of this work was to evaluate in dairy cows, the potential of ruminal degradability of four alfalfa (Medicago sativa) varieties developed in Argentina. The research was carried out in the Alfredo Volio Mata Experimental Station, located in the province of Cartago, Costa Rica; the area has a mean temperature of $17.9^{\circ} \mathrm{C}$; a height of 1542 masl, and $1465.9 \mathrm{~mm}$ of rain evenly distributed between the months of May and November. Sampling was performed during 2014's rainy season with ground level harvest of alfalfa, with samples of the varieties of Mora, Patricia, Victoria and Super Monarca, with harvest age of 28, 35, 42, 49, 56 and 63 days; all samples were dried, minced and incubated by duplicate on two Jersey-Reyna ruminally fistulated cows, for 0 , 3, 6, 12, 24 and 48 hours. The results were analyzed through the Marquardt algorithm with the SAS software. Statical differences were only found between soluble fraction and degradation rate $(\mathrm{p}<0.05)$ according to harvest ages, with values of soluble, potentially degradable and degradation rate of $35.34 \%-44.65 \%, 36.46 \%-39.96 \%$ and $0.0734 / \mathrm{h}-0.1176 / \mathrm{h}$, respectively.
\end{abstract}

1 Recibido: 10 de setiembre, 2016. Aceptado: 16 de enero, 2017. Este trabajo formó parte del proyecto de investigación VI-737-A8-129 “Innovación y desarrollo tecnológico de sistemas productivos caprinos" inscrito en Vicerrectoría de Investigación de la Universidad de Costa Rica, Costa Rica.

2 Universidad de Costa Rica, Facultad de Ciencias Agroalimentarias, Estación Experimental Alfredo Volio Mata. Cartago, Costa Rica. carlos. boschini@ucr.ac.cr, pablochaconh@ucr.ac.cr 
Likewise, effective degradability percentages of 59.06\%-67.09\%, with passage rates of $6 \% / \mathrm{h}$, according to harvest ages were obtained. Given the obtained results, the analyzed varieties of $M$. sativa showed a digestible forage, which could be used for the feeding of highly production animals.

Keywords: Medicago sativa, ruminant nutrition, in situ degradability.

\section{Introducción}

Los altos rendimientos de leche por vaca a base de pastos tropicales, no son nutricionalmente factibles, debido a que la cantidad de nutrientes disponibles en ellos no alcanza para el mantenimiento de altas producciones (Yari et al., 2012b). Por su parte, la alfalfa (Medicago sativa L.) es uno de los principales forrajes cultivados mundialmente (Yari et al., 2012a), porque tiene un mayor valor nutricional y alta degradabilidad cuando se compara con gramíneas o leguminosas tropicales de la misma edad (Jung y Engels, 2001). Por lo tanto, si la alfalfa es cosechada en condiciones adecuadas, presenta el potencial de reducir la necesidad de utilizar alimentos balanceados en las fincas ganaderas (Foster et al., 2007; Yari et al., 2012b).

Los sistemas de evaluación de alimentos para animales existentes, se enfocan en la dinámica de degradación de los constituyentes dietéticos (Balde et al., 1993; Trujillo et al., 2010). En el caso de la alfalfa, las características botánicas y su valor nutritivo se encuentran determinados por factores como el cultivar, la etapa de madurez y las condiciones climáticas de la zona donde se cultiva (Yari et al., 2012a).

La comprensión de la dinámica ruminal de los diferentes materiales utilizados en la fabricación de dietas, permitiría hacer un mejor uso de los recursos disponibles y reducir la generación de gases de efecto invernadero, nocivos sobre el ambiente (Hassanat et al., 2013).

Las leguminosas como la alfalfa tienen una amplia diversidad de tejidos, algunos de los cuales corresponden a células de pared gruesa, mientras que otros géneros tienen paredes celulares delgadas, existiendo también variaciones en el grado de lignificación de los tejidos (Jung y Engels, 2001); al mismo tiempo, el tipo y cantidad de uniones en las paredes celulares son determinantes del aprovechamiento de los contenidos celulares (Andrighetto et al., 1993).

Debido a que la calidad nutricional de la alfalfa se puede ver significativamente afectada, en primer lugar por la variedad cultivada, y en segundo término por las condiciones climáticas a las que se expone el cultivo y la ubicación geográfica de la parcela de siembra, se desarrolló el presente experimento en condiciones tropicales, con el fin de evaluar en bovinos de leche el potencial de la degradabilidad ruminal de cuatro variedades de alfalfa (Medicago sativa L.) originarias de la República Argentina.

\section{Materiales y métodos}

\section{Ubicación del experimento}

El experimento se llevó a cabo entre los meses de julio y diciembre de 2014, en la Estación Experimental Alfredo Volio Mata de la Universidad de Costa Rica, la cual se encuentra en El Alto de Ochomogo, distrito de San Rafael del cantón de La Unión en la provincia de Cartago, Costa Rica; ubicada a 1542 msnm. Las condiciones climatológicas comprenden una precipitación anual media de 1465,9 mm, distribuidos principalmente en la época lluviosa que va de mayo a noviembre; una humedad relativa media del $88 \%$ y una temperatura que va de los $13,5^{\circ} \mathrm{C}$ a los $22,2^{\circ} \mathrm{C}$ con un promedio anual de $17,9^{\circ} \mathrm{C}$ (IMN, comunicación personal, 2013). Las coordenadas geográficas 
correspondientes a dicho centro experimental son $9^{\circ} 55^{\prime} 10^{\prime \prime}$ latitud norte y 8357'20" longitud oeste (ChacónHernández y Boschini-Figueroa, 2016), el suelo es de origen volcánico, clasificado como Typic distrandepts, caracterizado por un buen drenaje y fertilidad media; asimismo, el ecosistema es considerado como bosque húmedo montano bajo (Vásquez, 1982).

\section{Descripción del material analizado}

El material analizado estuvo constituido por cuatro variedades de alfalfa desarrolladas por el Instituto Nacional de Tecnología Agropecuaria (INTA) de Argentina, todas con bajo grado de dormancia o latencia invernal (GRI). Las variedades evaluadas se denominan Victoria $(G R I=6)$, Patricia $(G R I=7)$, Súper Monarca $(G R I=8)$ y Mora (GRI $=9)$.

\section{Establecimiento de las variedades de alfalfa}

La siembra se realizó dos años y medio antes del inicio del periodo experimental. Alrededor de dos meses antes de la siembra, se aplicó una enmienda al terreno en forma de hidróxido de calcio, en una proporción de 2 t/ha con el fin de llevar el pH del suelo hasta un nivel mínimo de 6,2; posteriormente, al momento de la siembra, el suelo fue preparado por medio de pasadas respectivas de arado, rastra y rotador.

Las cuatro variedades se sembraron manualmente con una distancia entre surcos de $40 \mathrm{~cm}$ en parcelas de $1000 \mathrm{~m}^{2}$ cada una. Al momento de la siembra se aplicó fertilizante de fórmula química 10-30-10, en una dosis que asegurara una distribución de $90 \mathrm{~kg} /$ ha de $\mathrm{P}_{2} \mathrm{O}_{5}$. Junto con el fertilizante se aplicó un insecticida organofosforado cuyo ingrediente activo fue forato $10 \%$, distribuido en una cantidad de $15 \mathrm{~kg} / \mathrm{ha}$ de producto comercial. Posterior a la siembra, no se realizó ningún tipo de fertilización ni control químico de malezas, únicamente control cultural de arvenses.

\section{Diseño experimental}

Se utilizó un diseño irrestrictamente al azar con tratamientos en arreglo factorial (Steel y Torrie, 1988), donde se analizaron las cuatro variedades de alfalfa a seis edades de cosecha, correspondientes a tiempos de rebrote de 28 , 35, 42, 49, 56 y 63 días. La degradabilidad ruminal se llevó a cabo en dos vacas Jersey-Reyna fistuladas, siguiendo la metodología de Ørskov y McDonald (1979). Cada muestra analizada fue incubada ruminalmente por 0, 3, 6, 12, 24 y 48 horas. El modelo experimental utilizado para el análisis de los datos fue el siguiente:

$$
y_{i j k l}=\mu+V_{i}+E_{j}+e_{i j k l}
$$

donde:

$y=$ degradabilidad ruminal, $\%$

$\mu=$ promedio de degradabilidad

$V_{i}=$ efecto de la variedad

$E_{j}=$ efecto de la edad de cosecha

$e_{i j k l}=$ error experimental

\section{Proceso de muestreo y preparación de muestras}

Se realizaron muestreos durante el periodo lluvioso (agosto-setiembre) del año 2014, cuando cada variedad 
de alfalfa alcanzó las edades de rebrote evaluadas. Los muestreos consistieron en la cosecha al azar de tres puntos dentro de cada parcela de investigación; de cada lugar de muestreo se cortó el total de forraje disponible en un área de $1 \mathrm{~m}^{2}$ a una altura de $5 \mathrm{~cm}$ sobre el suelo. Posteriormente, la alfalfa cosechada se secó en un horno a $60{ }^{\circ} \mathrm{C}$ durante 48 horas, se molió a un tamaño de partícula de $3 \mathrm{~mm}$.

\section{Preparación y alimentación de los animales}

Los animales fistulados utilizados para la incubación de las muestras analizadas se mantuvieron en pastoreo y bajo una dieta estricta a base de pasto estrella africana (Cynodon nlemfluensis), desde quince días antes del inicio del periodo experimental, hasta el final del mismo.

\section{Análisis de laboratorio}

Los análisis fueron efectuados en el Laboratorio de Bromatología de la Estación Experimental Alfredo Volio Mata. Se realizaron pruebas de degradabilidad ruminal según la metodología descrita por Ørskov y McDonald (1979), para ajustar finalmente los resultados mediante el algoritmo de Marquardt (Vargas, 2005):

$$
Y=a+b\left(1-e^{c^{* t} t}\right)
$$

donde:

$\mathrm{Y}=$ degradación del material después de t horas de incubación, \%

$\mathrm{a}=$ fracción soluble, $\%$

$\mathrm{b}=$ fracción degradable, $\%$

$\mathrm{e}=$ base de los logaritmos neperianos

$\mathrm{c}=$ tasa de degradación instantánea, \%/h

$\mathrm{t}=$ tiempo de incubación ruminal en horas

A partir de los datos recolectados, se determinó también la degradabilidad efectiva de la alfalfa según la siguiente fórmula (Ørskov y McDonald, 1979):

$$
\mathrm{DE}=a+b \times c /(\mathrm{c}+\mathrm{k})
$$

donde:

$\mathrm{DE}=$ degradabilidad efectiva

$\mathrm{a}=$ fracción soluble, $\%$

$\mathrm{b}=$ fracción degradable, $\%$

$\mathrm{c}=$ tasa de degradación instantánea, $\% / \mathrm{h}$

$\mathrm{k}=$ tasa de pasaje, $\% / \mathrm{h}$

Como valor de k, se utilizó la velocidad de pasaje constante de $0,06 \% / \mathrm{h}$, recomendado para este tipo de materiales utilizados en la alimentación de vacas lecheras de alta producción (Coblentz y Hoffman, 2009; Cao et al., 2016). 


\section{Análisis de resultados}

Los valores determinados en laboratorio para las fracciones $\mathrm{Y}, \mathrm{a}, \mathrm{b}, \mathrm{c}, \mathrm{y}$ p en las muestras se sometieron al análisis de varianza con el PROC ANOVA del paquete estadístico SAS (2011). Aquellas variables que mostraron diferencias importantes entre medias, se les aplicó a la prueba de Duncan para su segregación estadística.

\section{Resultados y discusión}

Dentro de las constantes de degradabilidad evaluadas, las variedades de alfalfa estudiadas no mostraron diferencias significativas ( $>0,05$; Cuadro 1); similares observaciones han sido encontradas en el análisis de degradación in situ en los cultivares Pioneer y Beaver, para los cuales se reportaron valores de a, b y c de 41,8\%, $37,7 \%$ y $10,63 \% / \mathrm{h}$, respectivamente (Yu et al., 2004), porcentajes que son similares a los determinados en las muestras analizadas.

Cuadro 1. Constantes de degradabilidad ruminal de las cuatro variedades de alfalfa (Medicago sativa) provenientes del Instituto Nacional de Tecnología Agropecuaria de Argentina, cultivadas en la Estación Experimental Alfredo Volio Mata, Cartago, Costa Rica. 2014.

Table 1. Ruminal degradability constants of four Alfalfa (Medicago sativa) varieties brought from the National Agricultural Technology Institute in Argentina, grown at the Alfredo Volio Mata Experimental Station, Cartago, Costa Rica. 2014.

\begin{tabular}{lccccc}
\hline Variedad & $\mathbf{a}, \boldsymbol{\%}$ & $\mathbf{b}, \boldsymbol{\%}$ & $\mathbf{c}, \mathbf{h}$ & $\begin{array}{c}\text { Fracción potencialmente } \\
\text { degradable en el rumen, \% }\end{array}$ & $\begin{array}{c}\text { \% de degradabilidad } \\
\text { efectiva (k=0,06) }\end{array}$ \\
\hline Patricia & 41,73 & 38,66 & 0,1137 & 80,39 & 64,11 \\
Victoria & 39,81 & 38,53 & 0,0986 & 78,34 & 63,14 \\
Súper Monarca & 39,14 & 37,70 & 0,0976 & 76,84 & 62,94 \\
Mora & 38,43 & 37,16 & 0,0975 & 75,59 & 62,15 \\
\hline
\end{tabular}

a: fracción soluble, b: fracción potencialmente degradable, c: tasa de degradación de la fracción potencialmente degradable / a: soluble fraction, b: potentially degradable fraction, c: degradation rate of the potentially degradable fraction.

La digestibilidad in vitro de veintidós cultivares de alfalfa examinados por Broderick y Buxton (1991), mostró diferencias significativas, debido principalmente al origen genético del germoplasma utilizado; además, esas diferencias se produjeron cuando hubo una variabilidad en el origen genético de los ecotipos de alfalfa analizados. Según los mismos autores, cuando se evalúan materiales provenientes genéticamente de la especie Medicago sativa, no se espera que hayan diferencias entre variedades, puesto que únicamente se observan cuando se comparan con materiales que provienen de la especie Medicago falcata, por lo tanto, al ser las cuatro variedades analizadas derivadas de la especie $M$. sativa, no son esperables diferencias importantes entre ellas.

La digestibilidad in vitro de veintisiete variedades de alfalfa realizada por Tremblay et al. (2002), mostró diferencias significativas entre ellas, pero estas se debieron principalmente a las variaciones en la relación hoja:tallo de los cultivares, ya que la degradabilidad y la proporción del tallo son el principal determinante en el aprovechamiento de la alfalfa, esto debido a que usualmente los tallos tienen una cantidad de lignina mayor que el resto de la planta, incidiendo en la digestibilidad del alimento de forma importante (NRC, 2001).

A pesar de que la proporción de hojas y tallos en la presente investigación no fue determinada, la misma es dependiente de la variedad (Yu et al., 2004) y por lo tanto, se pueden especular similitudes en la relación de ambas 
fracciones y en la digestibilidad del tallo, siendo en parte responsables de la falta de diferencias estadísticamente significativas; aun así, más investigación al respecto se considera necesaria en las variedades analizadas.

Si se compara lo determinado en alfalfa con resultados de otros forrajes de uso común en ambientes tropicales, los valores de la fracción soluble encontrados en la presente investigación (Cuadro 1), fueron considerablemente mayores al 28,11\% reportado en morera a los 56 días de rebrote (Boschini, 2001). Asimismo, en el análisis de quince genotipos de sorgo forrajero, se reportó un máximo de 35,56\% encontrado en el CIAT 613 (Vargas, 2005), valores que también fueron menores a lo encontrado en las variedades de alfalfa evaluadas.

La mayor cantidad de componentes solubles en la alfalfa, indica una rápida disponibilidad de gran parte de los nutrientes contenidos en ese forraje, lo cual puede producir también un desacople en la disponibilidad de proteína y energía, principalmente al ser consumida por rumiantes (Broderick y Buxton, 1991; NRC, 2001). Por el contrario, la fracción potencialmente degradable de las variedades analizadas de alfalfa (Cuadro 1), fue menor a la que ha sido reportada anteriormente para Morus alba de 48,95\% (Martín et al., 2007) y sorgo negro forrajero (Sorghum almum). Para esta última variedad se encontró un 59,84\% potencialmente degradable al analizar la planta entera con ochenta días de rebrote (Boschini y Amador, 2001). Lo anterior indica que los componentes en la alfalfa analizada probablemente poseían una mayor cantidad de uniones entre los carbohidratos de la pared celular y compuestos fenólicos o lignina que la morera y el sorgo negro forrajero, ya que dichos enlaces están estrechamente relacionados con la disminución en la digestibilidad de la materia orgánica en rumiantes (Andrighetto et al., 1993).

En un estudio realizado por Naranjo y Cuartas (2011), los autores encontraron una tasa de degradación de 9\%/h en la morera, valor relativamente cercano a lo determinado en los materiales analizados (Cuadro 1); mientras que en quince genotipos de sorgo forrajero, Vargas (2005) encontró tasas de degradación muy variables, estando la mayoría por debajo del $4 \% / \mathrm{h}$, lo cual es considerablemente más bajo; esto último indica un aprovechamiento más veloz de las variedades de alfalfa estudiadas que de los sorgos investigados por Vargas, lo que también aceleraría su tasa de paso por el tracto gastrointestinal, permitiendo aumentar el consumo diario por el animal (NRC, 2001). Asimismo, Yu et al. (2004), reportaron valores de degradabilidad efectiva (DE) en alfalfa de 75,6\%, los cuales difieren de los presentados en el Cuadro 1; esa diferencia se da porque en la presente investigación se utilizó una tasa de paso por el rumen de $6 \% / \mathrm{h}$, mientras que en la investigación citada se empleó un valor de $4 \% / \mathrm{h}$. Al haber utilizado en la presente investigación una velocidad de paso más alta (para vacas de alta producción), la degradabilidad efectiva es menor, por el poco tiempo de residencia y fermentación permitido al material dentro del rumen.

En sorgo, en una investigación con quince variedades, la degradabilidad efectiva se encontró entre 43,17\% y 63,61\% (Vargas, 2005), siendo en términos generales, la mayoría de los valores reportados menores a lo determinado en las muestras analizadas, esto a pesar de que la tasa de paso utilizada en el sorgo fue un punto porcentual menor a la empleada en el análisis de la alfalfa. En morera, Kabi y Bareeba (2008) encontraron una proporción de degradabilidad efectiva en $M$. alba con una edad de corte de cuatro meses de $441,1 \mathrm{~g} / \mathrm{kg}(44,1 \%)$ que es cercano a lo determinado, a diferencia de esta investigación, los autores utilizaron una tasa de pasaje de 3\%/h, la cual es un 50\% más lenta a la considerada en el presente experimento, lo que indica que la morera requeriría más tiempo dentro del rumen para ser degradada en la misma proporción que la alfalfa analizada, y disminuye el potencial de consumo de los animales (NRC, 2001; CSIRO, 2007).

El valor de degradabilidad efectiva encontrado, indica un potencial de aprovechamiento de los nutrientes contenidos en la alfalfa y por ende, para ser empleada en la alimentación de animales altamente productivos (NRC, 2001).

La única fracción que mostró diferencias estadísticamente significativas $(\mathrm{p}<0,05)$ según la edad de cosecha de la alfalfa fue la fracción soluble (a), disminuyendo conforme aumentó el tiempo entre dos cortes sucesivos. Según los datos analizados, la diferencia en el aprovechamiento de la alfalfa por el animal corresponde a cambios en la cantidad de componentes solubles que se vuelven disponibles en los procesos digestivos y no por una disminución en la utilización de los nutrientes contenidos en la fracción degradable (Cuadro 2). 
Cuadro 2. Constantes de degradabilidad ruminal según la edad de rebrote en alfalfa (Medicago sativa), proveniente del Instituto Nacional de Tecnología Agropecuaria de Argentina, cultivada en la Estación Experimental Alfredo Volio Mata, Cartago, Costa Rica. 2014.

Table 2. Ruminal degradability constants according to growth age in alfalfa (Medicago sativa), brought from the National Agricultural Technology Institute in Argentina, grown at the Alfredo Volio Mata Experimental Station, Cartago, Costa Rica. 2004.

\begin{tabular}{|c|c|c|c|c|c|}
\hline $\begin{array}{c}\text { Edad de } \\
\text { cosecha, días }\end{array}$ & a, $\%$ & b, $\%$ & $\mathbf{c}, / \mathbf{h}$ & $\begin{array}{c}\text { Fracción potencialmente } \\
\text { degradable en el rumen, \% }\end{array}$ & $\begin{array}{c}\% \text { de degradabilidad } \\
\text { efectiva }(k=0,06)\end{array}$ \\
\hline 28 & $44,65 \mathrm{~d}$ & 36,46 & 0,1068 & $81,11 \mathrm{~d}$ & $67,09 \mathrm{~d}$ \\
\hline 35 & 40,62 de & 38,56 & 0,1115 & 79,18 de & 65,39 de \\
\hline 42 & $43,28 \mathrm{~d}$ & 37,75 & 0,0734 & $81,03 \mathrm{~d}$ & 63,71 e \\
\hline 49 & 37,28 ef & 39,96 & 0,1176 & 77,24 ef & 63,33 e \\
\hline 56 & 37,50 ef & 37,14 & 0,1011 & $74,64 \mathrm{gf}$ & $59,92 \mathrm{f}$ \\
\hline 63 & $35,34 \mathrm{f}$ & 38,22 & 0,1006 & $73,56 \mathrm{~g}$ & $59,06 \mathrm{f}$ \\
\hline
\end{tabular}

a: fracción soluble, b: fracción potencialmente degradable, c: tasa de degradación de la fracción potencialmente degradable / a: soluble fraction, b: potentially degradable fraction, c: degradation rate of the potentially degradable fraction.

$\mathrm{d}$, e, f, $\mathrm{g}$ indican diferencias significativas entre edades de cosecha $(\mathrm{p}<0,05) / \mathrm{d}$, e, $\mathrm{f}$, g show significant differences among growth ages $(\mathrm{p}<0.05)$.

Como ha sido reportado en otras investigaciones, las ventajas de la alfalfa en términos de degradabilidad, se deben a su nivel de contenidos celulares y a la velocidad de degradación de la fracción degradable (Andrighetto et al., 1993), por lo tanto, con el fin de explotar al máximo esta característica, el material se aprovecha más por los rumiantes conforme se disminuye la edad de cosecha a la que son utilizadas las distintas variedades.

Similar a lo determinado en la presente investigación (Cuadro 2), se ha encontrado una disminución en la materia seca potencialmente degradable de la alfalfa al aumentar la edad de cosecha, desde un máximo de 83,1\% al inicio de la etapa de floración hasta 70,5\% al final de la etapa reproductiva (Balde et al., 1993). Canbolat et al. (2006), también determinaron una disminución de la fracción potencialmente degradable en el rumen conforme se aumentó la edad de cosecha hasta el valor máximo de 73,70\% antes de que se inicie la floración, siendo ese valor similar a lo determinado a los 63 días de rebrote en las muestras analizadas.

En la materia orgánica de un heno de alfalfa cosechado a setenta días de rebrote, Yari et al. (2012b), encontraron un $13,0 \%$ soluble, $14,0 \%$ potencialmente degradable y $47,5 \%$ de digestibilidad efectiva, valores que son menores a los determinados en la presente investigación (Cuadros 1 y 2), esas diferencias podrían ser atribuidas a que, en el experimento de Yari et al. (2012b) no se tomó en cuenta la fracción inorgánica presente en la alfalfa y que lógicamente, de ser tomada en cuenta dentro de los cálculos, producen una variación importante en los resultados, debido al contenido de cenizas que normalmente existe en la alfalfa. Adicionalmente, según Yari et al. (2012b), la edad de cosecha es un factor determinante de la calidad nutricional de la alfalfa y por lo tanto, la mayor edad de cosecha que evaluaron esos autores hizo que el material se encontrara en otra etapa fisiológica, transformándose en otra razón por la cual el material tiene valores menores a los presentados.

El valor determinado de degradabilidad potencial de la alfalfa (Figura 1 y Cuadro 2), puede ser explicado por la alta digestibilidad de las uniones de los ácidos ferúlico y p-cumárico en la pared celular, los cuales después de 72 horas de incubación en el rumen presentan un $89 \%$ y $82 \%$ de digestión en el rumen, respectivamente, indicando una más fácil digestión de la fibra por los microorganismos (Cao et al., 2016). 


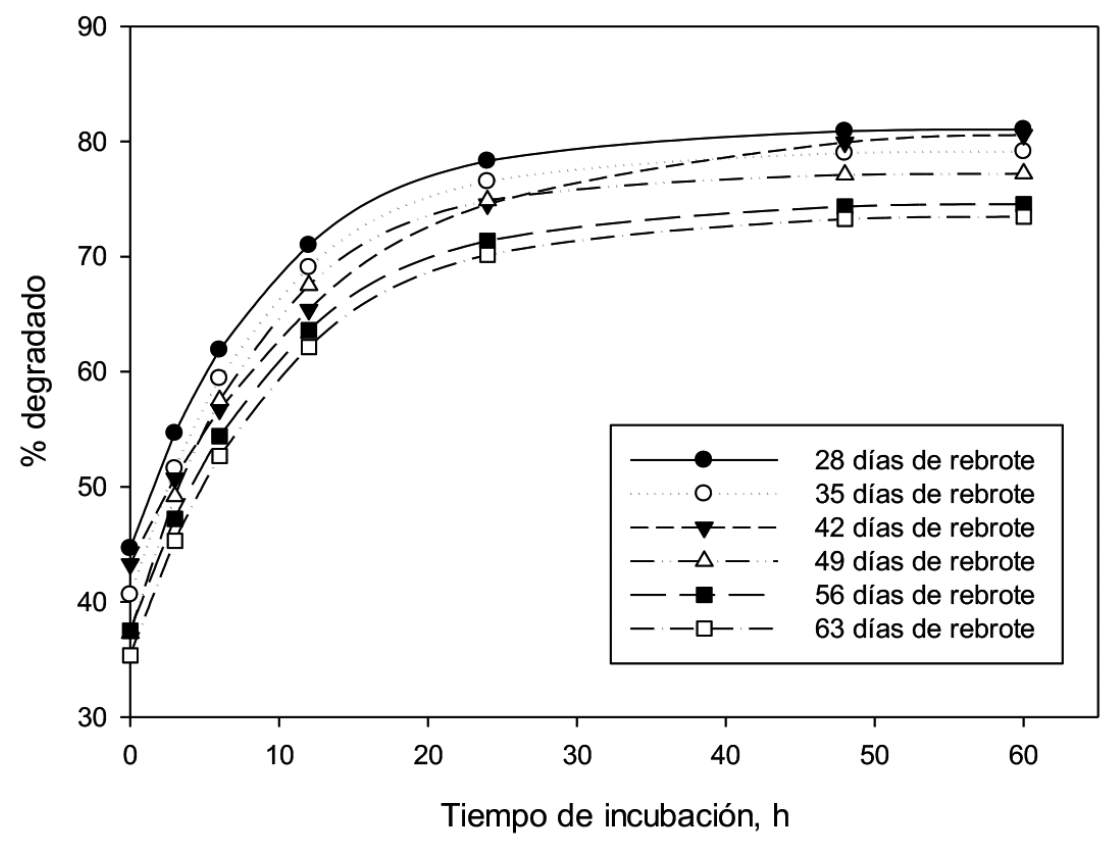

Figura 1. Curva de degradabilidad ruminal según la edad de rebrote de la alfalfa, proveniente del Instituto Nacional de Tecnología Agropecuaria de Argentina, cultivada en la Estación Experimental Alfredo Volio Mata, Cartago, Costa Rica. 2014.

Figure 1. Ruminal degradability curve according to growth age of alfalfa, brought from the National Agricultural Technology Institute in Argentina, grown at the Alfredo Volio Mata Experimental Station, Cartago, Costa Rica. 2014.

Un valor de degradabilidad efectiva de $41 \%$ ha sido reportado en heno de alfalfa cosechada a la mitad de la floración con tasas de paso de 6\%/h (Trujillo et al., 2010), lo cual es considerablemente menor a lo determinado en las muestras analizadas (Cuadro 2), además de esa diferencia en la degradabilidad efectiva, Coblentz et al. (1998) consideran inapropiada la asignación de una tasa de pasaje fija a los forrajes para estimar la degradabilidad efectiva, razón por la cual, ambas estimaciones podrían estar sesgadas.

\section{Conclusiones}

En términos generales, se puede observar como la alfalfa constituye un forraje de alta calidad y con un muy buen potencial de aprovechamiento por los rumiantes, inclusive por sobre otros forrajes de uso común en ambientes tropicales; por lo tanto, la posibilidad de introducir este tipo de materiales en la dieta de animales de alta producción, favorecería el balance nutricional de las dietas tropicales, las cuales se caracterizan por estar conformadas por materiales de bajo valor (Blache et al., 2008; Chacón-Hernández y Boschini-Figueroa, 2016). 


\section{Agradecimientos}

Los autores expresan su agradecimiento al Dr. Daniel Basigalup del INTA Manfredi, Argentina y al Ing. Enrique Linares de Produsem S.A., por la colaboración en la selección y obtención de la semilla de alfalfa de las variedades evaluadas.

\section{Literatura citada}

Andrighetto, I., L. Bailoni, G. Cozzi, H.F. Tolosa, B. Hartman, M. Hinds, and D. Sapienza. 1993. Observations on in situ degradation of forage cell components in Alfalfa and Italian Ryegrass. J. Dairy Sci. 76:2624-2631.

Balde, A.T., J.H. Vandersall, R.A. Erdman, J.B. Reeves-III, and B.P. Glenn. 1993. Effect of stage of maturity of alfalfa and orchardgrass on in situ dry matter and crude protein degradability and amino acid composition. Anim. Feed Sci. Technol. 44:29-43.

Blache, D., S.K. Maloney, and D.K. Revell. 2008. Use and limitations of alternative feed resources to sustain and improve reproductive performance in sheep and goats. Anim. Feed Sci. Technol. 147:140-157.

Boschini, C. 2001. Degradabilidad in situ de la materia seca, proteína y fibra del forraje de morera (Morus alba). Agron. Mesoam. 12:79-87.

Boschini, C., y A.L. Amador. 2001. Degradabilidad ruminal de la planta de sorgo negro forrajero (Sorghum almum) en diferentes etapas de crecimiento. Agron. Mesoam. 12:169-174.

Broderick, G.A., and D.R. Buxton. 1991. Genetic variation in alfalfa for ruminal protein degradability. Can. J. Anim. Sci. 71:755-760

Canbolat, O., A. Kamalak, C.O. Ozkan, A. Erol, M. Sahin, E. Karakas, and E. Ozkose. 2006. Prediction of relative feed value of alfalfa hays harvested at different maturity stages using in vitro gas production. Liv. Res. Rural Dev. 18:1-8.

Cao, B.B., R. Wang, Y.K. Bo, S. Bai, and H.J. Yang. 2016. In situ rumen digestibility of ester-linked ferulic and p-coumaric acids in crop stover or straws in comparison with alfalfa and Chinese wild ryegrass. Anim. Feed Sci. Technol. 212:27-34.

Coblentz, W.K., J.O. Fritz, W.H. Fick, R.C. Cochran, and J.E. Shirley. 1998. In situ dry matter, nitrogen, and fiber degradation of alfalfa, red clover, and eastern gamagrass at four maturities. J. Dairy Sci. 81:150-161.

Coblentz, W.K., and P.C. Hoffman. 2009. Effects of bale moisture and bale diameter on spontaneous heating, dry matter recovery, in vitro true digestibility, and in situ disappearance kinetics of alfalfa-orchardgrass hays. J. Dairy Sci. 92:2853-2874.

CSIRO (Commonwealth Scientific and Industrial Research Organisation). 2007. Nutrient requirements of domesticated ruminants. CSIRO Publishing, AUS.

Chacón-Hernández, P., y C. Boschini-Figueroa. 2016. Crecimiento del ganado caprino en una finca del Valle Central de Costa Rica. Agron. Mesoam. 27:159-165.

Foster, J.L., J.P. Muir, B.D. Lambert, and D. Pawelek. 2007. In situ and in vitro degradation of native Texas warm-season legumes and alfalfa in goats and steers fed a sorghum-sudan basal diet. Anim. Feed Sci. Technol. 133:228-239.

Hassanat, F., R. Gervais, C. Julien, D.I. Massé, A. Lettat, P.Y. Chouinard, H.V. Petit, and C. Benchaar. 2013. Replacing alfalfa silage with corn silage in dairy cow diets: Effects on enteric methane production, ruminal fermentation, digestion, $\mathrm{N}$ balance, and milk production. J. Dairy Sci. 96:4553-4567.

Jung, H.G., and F.M. Engels. 2001. Alfalfa stem tissues: rate and extent of cell-wall thinning during ruminal degradation. Neth. J. Agric. Sci. 49:3-13. 
Kabi, F., and F.B. Bareeba. 2008. Herbage biomass production and nutritive value of mulberry (Morus alba) and Calliandra calothyrsus harvested at different cutting frequencies. Anim. Feed Sci. Technol. 140:178-190.

Martín, G.J., Y. Noda, G. Pentón, D.E. García, F. García, E. González, F. Ojeda, M. Milera, O. López, J. Ly, L. Leiva, and J. Arece. 2007. La morera (Morua alba, Linn.): una especie de interés para la alimentación animal. Pastos y Forrajes 30:1-12.

NRC (National Research Council). 2001. Nutrient requirements of dairy cattle. $7^{\text {th }}$ ed. National Academy Press, WA, USA.

Naranjo, J.F., y C. Cuartas. 2011. Caracterización nutricional y de la cinética de degradación ruminal de algunos de los recursos forrajeros con potencial para la suplementación de rumiantes en el trópico alto de Colombia. Rev. CES Med. Vet. Zootec. $6(1): 9-19$.

Ørskov, E.R., and I. McDonald. 1979. The estimation of protein degradability in the rumen from incubation measurements weighted according to rate of passage. J. Agric. Sci. (Camb.) 92:499-503.

SAS. 2011. SAS/STAT® 9.3 User's guide. SAS Institute Inc, Cary, NC. USA.

Steel, R., y J. Torrie. 1988. Bioestadística: principios y procedimientos. McGraw Hill, México DF, MEX.

Tremblay, G.F., G. Bélanger, K.B. McRae, and R. Michaud. 2002. Leaf and stem dry matter digestibility and ruminal undegradable proteins of alfalfa cultivars. Can. J. Anim. Sci. 82:383-393.

Trujillo, A.I., M.D. Marichal, and M. Carriquiry. 2010. Comparison of dry matter and neutral detergent fibre degradation of fibrous feedstuffs as determined with in situ and in vitro gravimetric procedures. Anim. Feed Sci. Technol. 161:49-57.

Vargas, C.F. 2005. Valoración de parámetros nutricionales y degradabilidad ruminal de 15 ecotipos de sorgo forrajero (Sorghum sp.). Agron. Mesoam. 16:215-223.

Vásquez, A. 1982. Estudio detallado de los suelos de la Estación Experimental de Ganado Lechero El Alto. Universidad de Costa Rica, San José, CRC.

Yari, M., R. Valizadeh, A.A. Naserian, G.R. Ghorbani, and P.R. Moghaddam. 2012a. Botanical traits, protein and carbohydrate fractions, ruminal degradability and energy contents of alfalfa hay harvested at three stages of maturity and in the afternoon and morning. Anim. Feed Sci. Technol. 172:162-170.

Yari, M., R. Valizadeh, A.A. Naserian, A. Jonker, and P. Yu. 2012b. Modeling nutrient availability of alfalfa hay harvested at three stages of maturity and in the afternoon and morning in dairy cows. Anim. Feed Sci. Technol. 178:12-19.

Yu, P., D.A. Christensen, and J.J. McKinnon. 2004. In situ rumen degradation kinetics of timothy and alfalfa as affected by cultivar and stage of maturity. Can. J. Anim. Sci. 84:255-263. 